\title{
Cognitively-Inspired Computing for Gerontechnology
}

\author{
Antonio Fernández-Caballero $^{1} \cdot$ Pascual González $^{1} \cdot$ Elena Navarro $^{1}$
}

Published online: 11 March 2016

(c) Springer Science+Business Media New York 2016

Gerontechnology [1] is an interdisciplinary field combining gerontology and technology. Sustaining an ageing society depends upon our effectiveness in creating intelligent systems, using assistive technology and inclusive design, to help older adults enjoy innovative and independent living and participate socially in conditions of good health, comfort and safety. Gerontechnology aims to match systems to the health, housing, mobility, communication, leisure and work of older people.

The development of these systems is a challenging activity involving disciplines as diverse as Artificial Intelligence, Human-Computer Interaction, Neurobiology, Cognitive Psychology and Engineering to work together in order to provide solutions to satisfy this growing demand of the society. Stakeholders must be able to create systems that are intelligent enough to detect falls or emotions, userfriendly enough to be managed by people with mobility and cognitive problems, and reliable and autonomous enough to monitor health and facilitate mobility.

This Special Issue focuses on all aspects of cognitive agents, addressed by current practices and future trends in Gerontechnology. These include perception, action, affective and cognitive learning and memory, attention, decision making and control, social cognition, language processing and communication, reasoning, problem solving, and consciousness.

The Special Issue on "Cognitively-Inspired Computing for Gerontechnology" attracted numerous papers of the highest quality, of which five were finally accepted.

Antonio Fernández-Caballero

antonio.fdez@uclm.es

1 Instituto de Investigación en Informática de Albacete, Universidad de Castilla-La Mancha, 02071 Albacete, Spain
Moreover, in order to ensure a fair review process, the final articles, co-authored by the guest editors, were reviewed independently by another editorial board member. The articles that make up this Special Issue cover most of the initial topics outlined during the call for papers, namely, cognitively-inspired computing for assistive technologies and devices; cognitively-inspired computing for household accident detection; emotion/affect/mood recognition and regulation; personalised ambient adaptation; social/care cognitive agents; intelligent telehealth, telemedicine and communication services; social networks for the elderly; lifelong learning for mental health.

The first paper included in the Special Issue is authored by Kötteritzsch and Weyers [4]. The authors present a review of the literature on assistive technologies for older adults in urban areas. The goal of their contribution is to analyse the impact of Ambient Assisted Living systems on urban areas and identify challenges for future research. The authors review the literature on assistive technology of the past decade, focusing on its potential application in urban areas, and propose a classification of the approaches presented. The article discusses future research, with challenges emerging from novel network technologies, market uptake or adaptation, and the support of social neighbourhood structures.

In the next article, Rodríguez et al. [6] present an interactive fuzzy inference system for teletherapy of older people. This paper proposes a system enabling therapists to create bespoke motor therapies for ageing people as state diagrams and manage them efficiently in a collaborative setting. The proposed system is equipped with a fuzzybased decision-making component that therapists can use to design the control for transitioning between states according to variables such as fatigue and performance. The system makes it feasible to provide older patients with 
the treatment they need in their own homes while effectiveness is controlled by a fuzzy inference system.

The third article, written by Griol and Callejas [3], describes a framework that allows developing contextaware multimodal conversational agents that dynamically incorporate user-specific requirements and preferences as well as characteristics of the interaction environment, in order to improve and personalise the service provided. Their proposal integrates the facilities of the Android API in a modular architecture that emphasises interaction management and context-awareness to build user-adapted, robust and maintainable applications. As a proof of concept, the authors have used the proposed framework to develop an Android app for older adults with Alzheimer's disease.

The next article, authored by Castillo et al. [2], introduces the architecture of a project devoted to emotionaware Ambient Intelligence and Gerontechnology, which uses emotion regulation techniques to find solutions for improving the quality of life and care of the ageing adult who can or wants to continue living at home. A series of sensors is used for monitoring the older adults' facial and gestural expressions, activity and behaviour, as well as relevant physiological data. In this way, their emotions are inferred and recognised. Music, colour and light are the means of stimulation used to regulate their emotions towards a positive and pleasant mood.

In the last article presented of this Special Issue, Pires et al. [5] propose an integrated e-health care system for the support of older adults. The system enables the biomedical parameters of a person to be monitored in real time, anywhere and in any situation with no interference to his/her daily routines. The system comprises a personal biomedical data acquisition subsystem and an information storage centre. The sensory devices developed are responsible for acquiring and wirelessly transmitting the biomedical signals to a smartphone or tablet. The collected information can be managed and maintained in the storage centre. To help ensure well-founded diagnoses, the medical data are accessible to the organisations responsible for the patients' medical records.

The guest editors would like to thank the anonymous reviewers for their valuable and timely reviews of the manuscripts, which have helped to ensure the quality of this Special Issue. We would also thank the editor-in-chief for his very valuable recommendations during the whole editorial process.

\section{References}

1. Bouma H, Fozard JL, Bouwhuis DG, Taipale VT. Gerontechnology in perspective. Gerontechnology. 2007;6:190-216.

2. Castillo JC, Castro-González Á, Fernández-Caballero A, Latorre JM, Pastor JM, Fernández-Sotos A, Salichs MA. Software architecture for smart emotion recognition and regulation of the ageing adult. Cogn Comput. 2016. doi:10.1007/s12559-016-9383y.

3. Griol D, Callejas Z. Mobile conversational agents for contextaware care applications. Cogn Comput. 2016. doi:10.1007/s12559015-9352-x.

4. Kötteritzsch A, Weyers B. Assistive technologies for older adults in urban areas: a literature review. Cogn Comput. 2016. doi:10. 1007/s12559-015-9355-7.

5. Pires P, Mendes L, Mendes J, Rodrigues R, Pereira A. Integrated e-health care system for elderly support. Cogn Comput. 2016. doi:10.1007/s12559-015-9367-3.

6. Rodríguez AC, Roda C, Montero F, González P, Navarro E. An interactive fuzzy inference system for teletherapy of older people. Cogn Comput. 2016. doi:10.1007/s12559-015-9356-6. 